Supporting information

\title{
In Situ Sulfidation for Controllable Heterointerface of Cobalt Oxides-Cobalt Sulfides on 3D Porous Carbon Realizing Efficient Rechargeable Liquid/Solid-State Zn-Air Batteries
}

Wen-Wen Tian, Jin-Tao Ren, Xian-Wei Lv, Li-Jiao Gao, and Zhong-Yong Yuan*

Key Laboratory of Advanced Energy Materials Chemistry (Ministry of Education),

School of Materials Science and Engineering, Nankai University, Tianjin 300350, China.

*Corresponding author. E-mail address: zyyuan@nankai.edu.cn (Z.-Y. Yuan)

12 Pages

9 Figures

2 Tables 

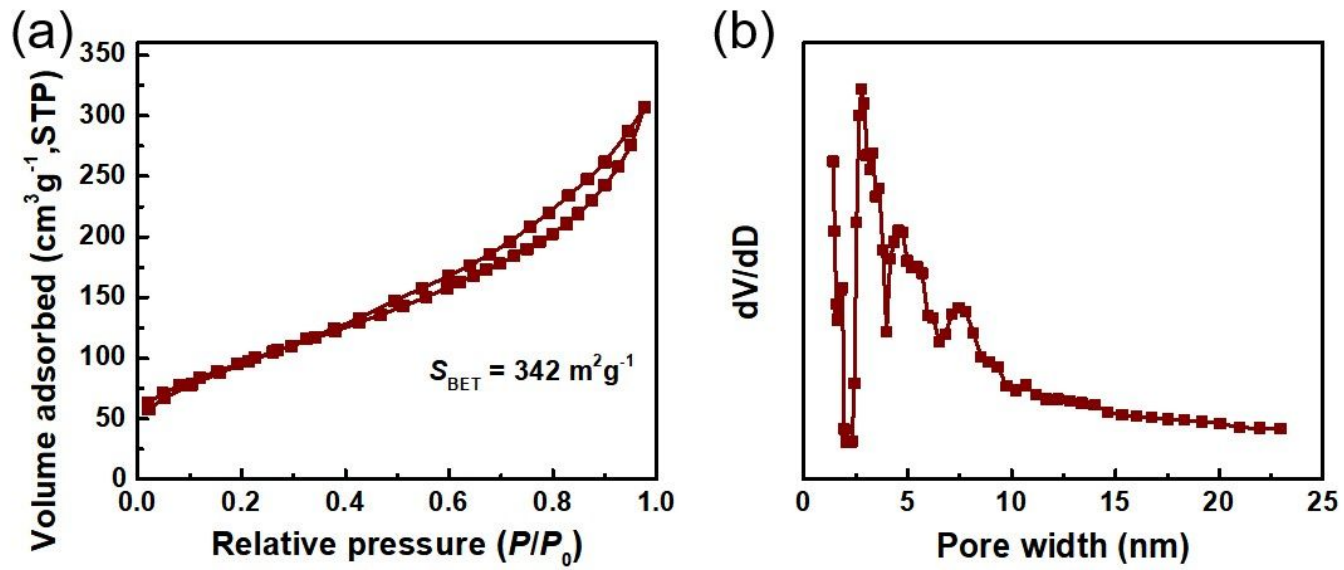

Figure S1. (a) $\mathrm{N}_{2}$ adsorption-desorption isotherms of Co-S-O/NSCN and (b) the corresponding pore-size distribution curve.

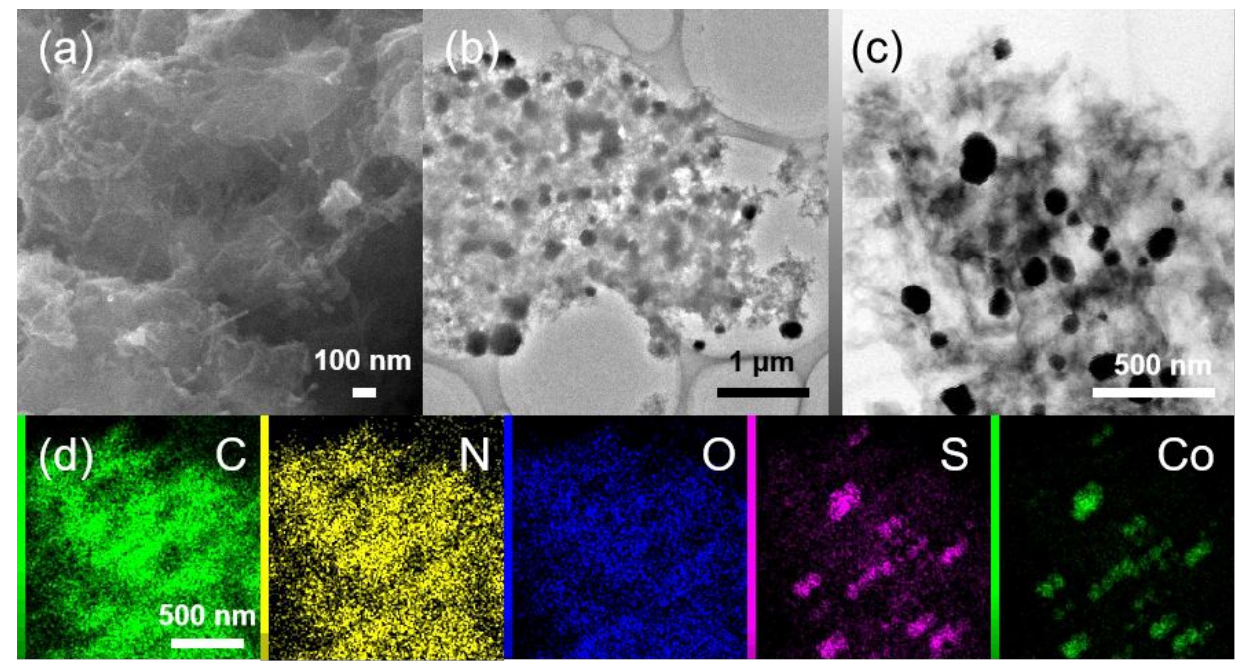

Figure S2. (a) SEM and (b) TEM images of Co-S/NSCN. (c) BF-STEM and (d) corresponding TEM-EDX elemental mappings of Co-S/NSCN. 


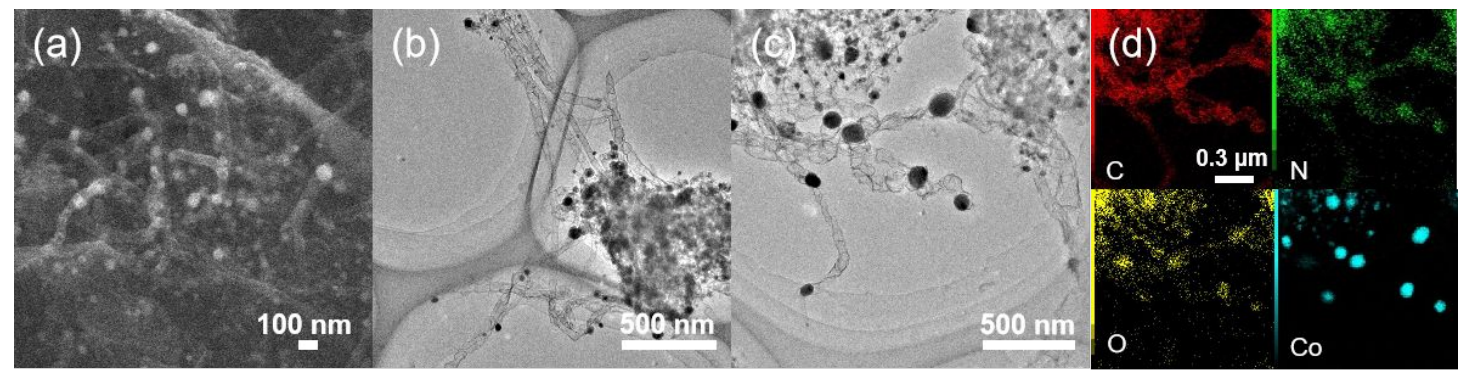

Figure S3. (a) SEM and (b-c) TEM images of Co@NCNTs/NCS. (d) TEM-EDX elemental mappings of $\mathrm{Co} @ \mathrm{NCNTs} / \mathrm{NCS}$.
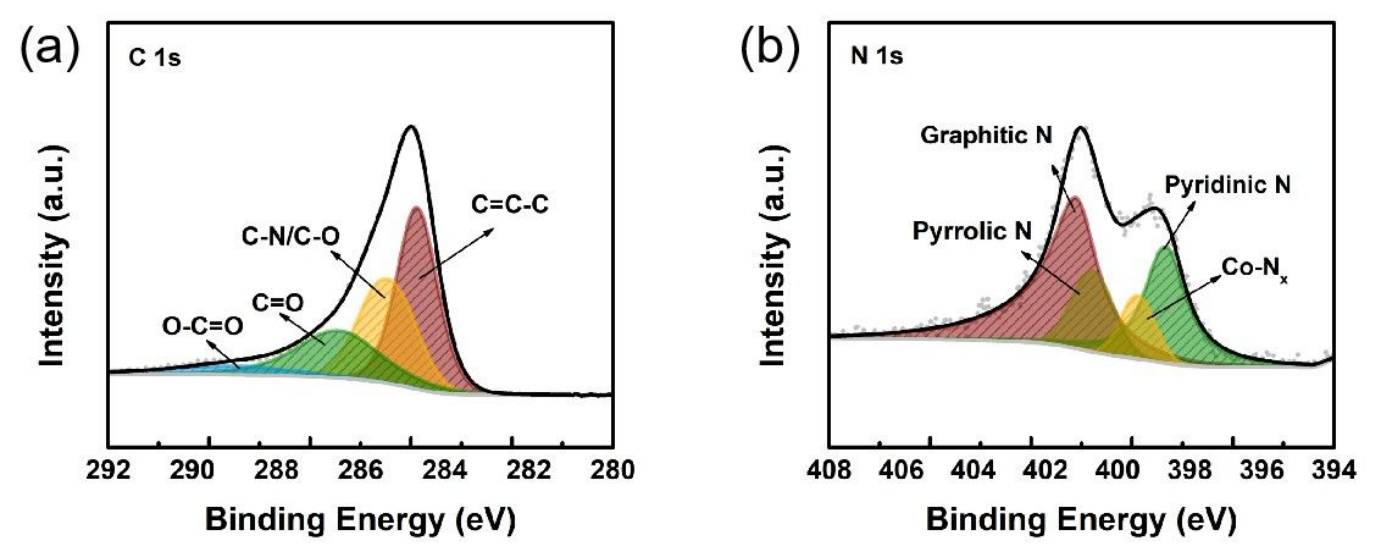

Figure S4. High-resolution of (a) C 1s and (b) N 1s XPS spectra for Co-S-O/NSCN.
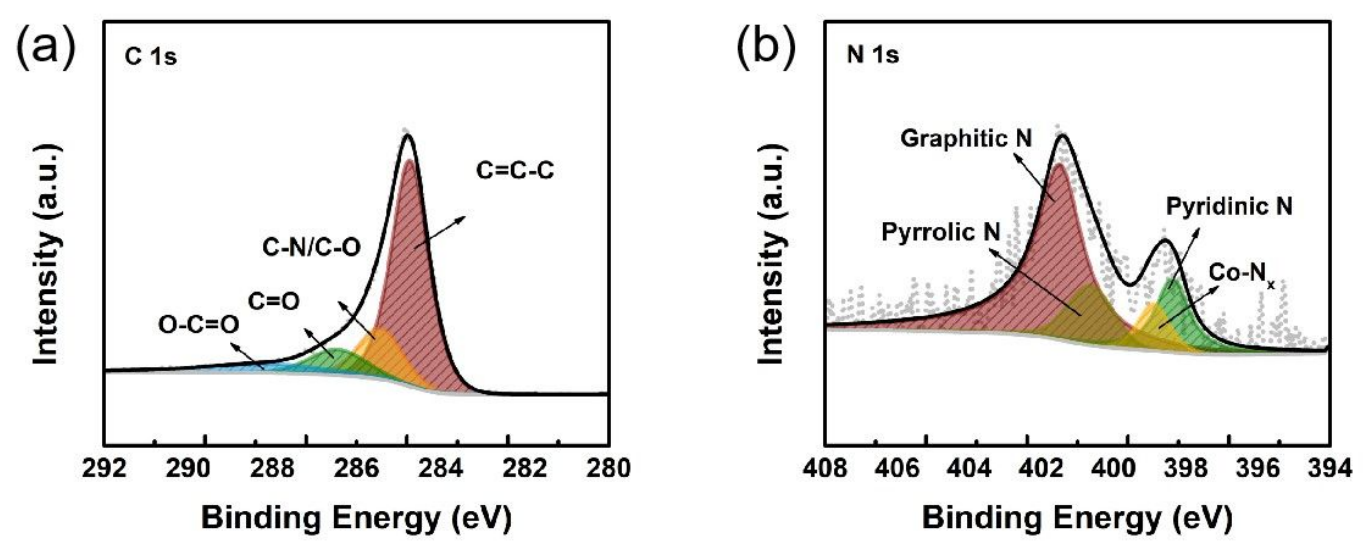

Figure S5. High-resolution of (a) C 1s and (b) N 1s XPS spectra for Co-S/NSCN. 

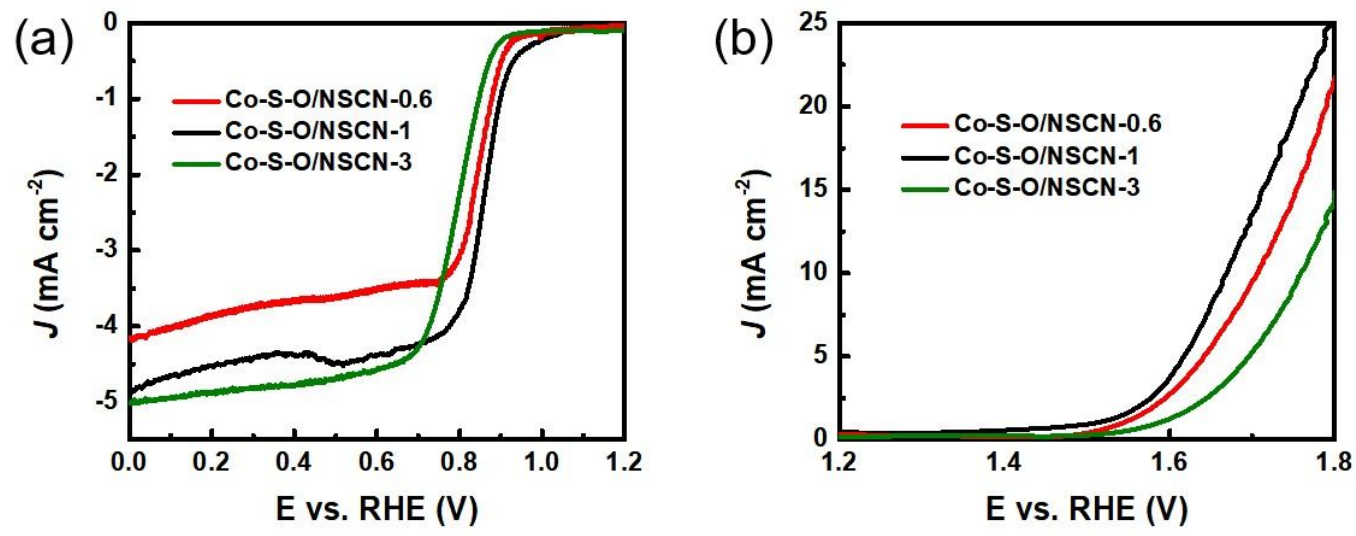

Figure S6. (a) ORR polarization curves and (b) OER polarization curves of Co-S-O/NSCN catalysts with cobalt addition amount of $0.6,1$ and $3 \mathrm{mmol}$ in $\mathrm{O}_{2}$-saturated $0.1 \mathrm{M} \mathrm{KOH}$.
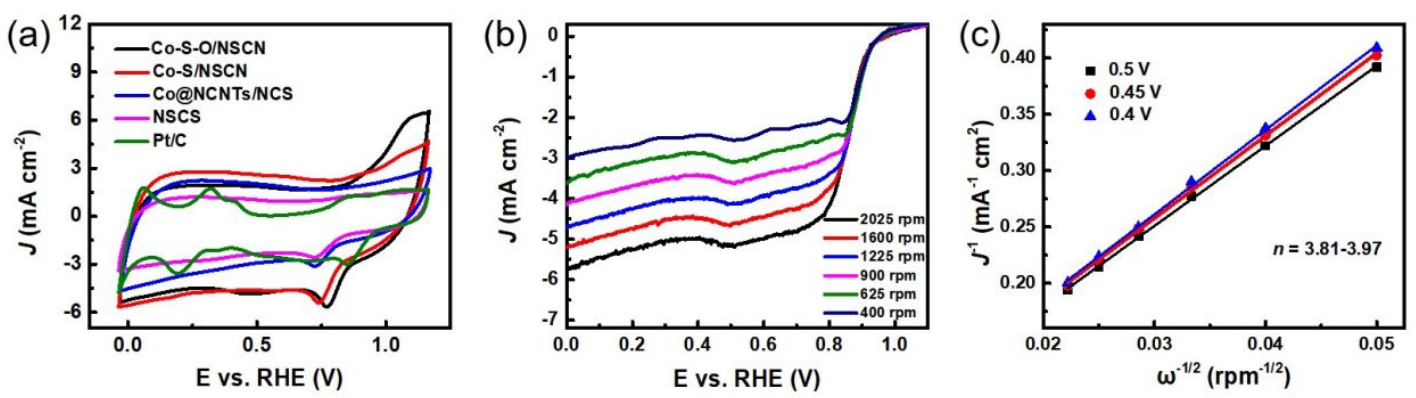

Figure S7. (a) CV curves of different catalysts in $\mathrm{O}_{2}$-saturated $0.1 \mathrm{M} \mathrm{KOH}$. (b) LSV curves under various rotating speeds from 2025 to $400 \mathrm{rpm}$ for Co-S-O/NSCN. (c) The corresponding K-L plots at 0.4-0.5 V for Co-S-O/NSCN. 

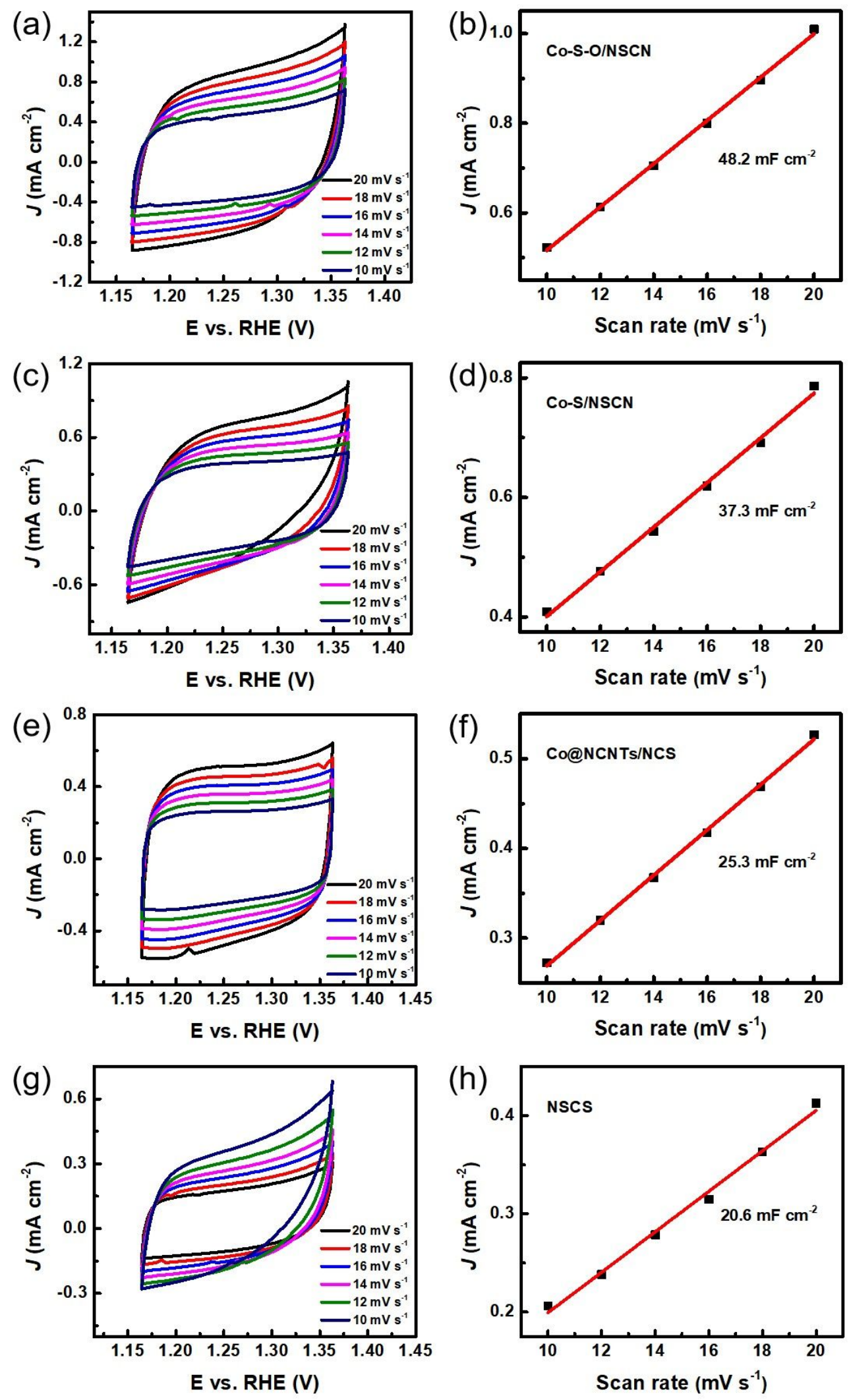

Figure S8. CV curves at different scan rates from 10 to $20 \mathrm{mV} \mathrm{s}^{-1}$ and the corresponding current densities against scan rates at $1.30 \mathrm{~V}$ (vs. RHE) of obtained catalysts. (a,b) Co-S-O/NSCN, (c,d) Co-S/NSCN, (e,f) Co@NCNTs/NCS and (g,h) NSCS. 

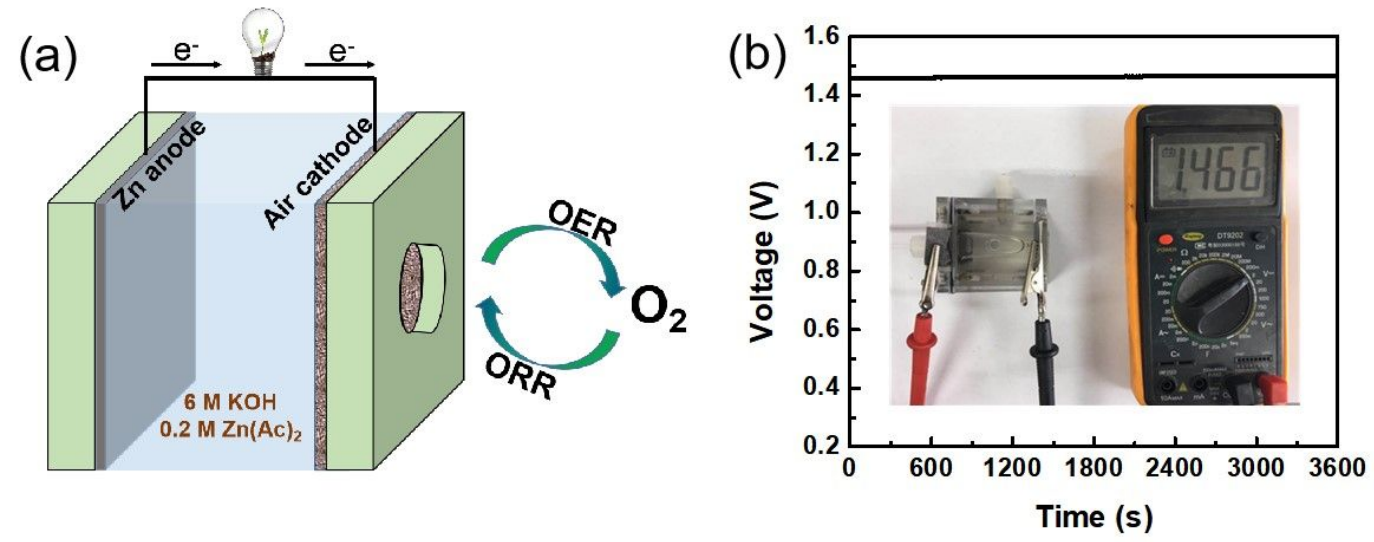

Figure S9. (a) Schematic illustration of the two-electrode rechargeable Zn-air battery. (b) Plots of the open-circuit potential against time of the fabricated $\mathrm{Zn}$-air batteries assembled by $\mathrm{Co}-\mathrm{S}-\mathrm{O} / \mathrm{NSCN}$, the inset of (b) is optical photograph of the open-circuit potential of the Co-S-O/NSCN assembled Zn-air battery. 
Table S1. Comparison of the electrocatalytic activity of recently reported bifunctional Co-based electrocatalysts in alkaline electrolyte.

\begin{tabular}{|c|c|c|c|c|c|}
\hline Catalyst & $\begin{array}{l}E_{\text {onset }} /(\mathbf{V} \\
\text { vs. RHE) }\end{array}$ & $\begin{array}{c}E_{1 / 2} /(\mathrm{V} \text { vs. } \\
\text { RHE) }\end{array}$ & $\begin{array}{c}E_{\mathrm{j}=10} /(\mathrm{V} \text { vs. } \\
\text { RHE) }\end{array}$ & $\begin{array}{c}E_{\text {gap }} /(\mathrm{V} \text { vs. } \\
\text { RHE) }\end{array}$ & Ref. \\
\hline $\mathrm{Co}-\mathrm{S}-\mathrm{O} / \mathrm{NSCN}$ & 0.950 & 0.865 & 1.665 & 0.8 & $\begin{array}{l}\text { This } \\
\text { work. }\end{array}$ \\
\hline Co-N carbon nanocage & 0.92 & 0.82 & 1.64 & 0.82 & [1] \\
\hline $\mathrm{Co}_{9} \mathrm{~S}_{8} / \mathrm{NSPC} 9-45$ & 0.90 & 0.74 & 1.57 & 0.83 & [2] \\
\hline $\mathrm{Co} @ \mathrm{Co}_{3} \mathrm{O}_{4} / \mathrm{NC}$ & 0.91 & 0.8 & 1.65 & 0.85 & [3] \\
\hline $\mathrm{CoO} / \mathrm{N}$-doped graphene & 0.90 & 0.81 & 1.57 & 0.76 & [4] \\
\hline $\mathrm{NiCo} / \mathrm{PFC}$ aerogels & 0.92 & 0.76 & 1.62 & 0.86 & [5] \\
\hline $\mathrm{Co} / \mathrm{S} / \mathrm{N}-800$ & 0.912 & 0.831 & 1.591 & 0.76 & [6] \\
\hline Co-Ni-S@NSPC & 0.95 & 0.82 & 1.7 & 0.88 & [7] \\
\hline $\mathrm{Co}-\mathrm{N} / \mathrm{C} 800$ & N.A. & 0.65 & 1.64 & 0.99 & [8] \\
\hline $\mathrm{Co}_{9} \mathrm{~S}_{8} \mathrm{HMs}-140$ & 0.95 & 0.82 & 1.65 & 0.83 & [9] \\
\hline $\mathrm{CoFe}_{2} \mathrm{O}_{4} @ \mathrm{CNTs}$ & N.A. & 0.78 & 1.74 & 0.96 & {$[10]$} \\
\hline $\mathrm{FeCo}-\mathrm{N} / \mathrm{C}$ & 0.98 & 0.84 & 1.61 & 0.77 & {$[11]$} \\
\hline $\mathrm{Co}_{9} \mathrm{~S}_{8} @ \mathrm{MoS}_{2}$ & N.A. & 0.884 & 1.67 & 0.786 & [12] \\
\hline CoO@Co/N-rGO & 0.95 & 0.85 & 1.67 & 0.82 & {$[13]$} \\
\hline $\mathrm{Co}_{0.85} \mathrm{Se} @ \mathrm{NC}$ & 0.917 & 0.82 & 1.76 & 0.94 & {$[14]$} \\
\hline CoP@CC & 0.80 & 0.67 & 1.68 & 1.01 & [15] \\
\hline
\end{tabular}


Table S2. The performance comparison of the present reported liquid rechargeable $\mathrm{Zn}$-air battery.

\begin{tabular}{|c|c|c|c|c|}
\hline Catalyst & $\begin{array}{l}\text { Loading } \\
\text { mass }(\mathbf{m g} \\
\left.\mathbf{c m}^{-2}\right)\end{array}$ & $\begin{array}{c}\text { Peak power } \\
\text { density }(\mathbf{m W} \\
\left.\mathbf{c m}^{-2}\right)\end{array}$ & $\begin{array}{c}\text { Cycling conditions and } \\
\text { stability }\end{array}$ & Ref. \\
\hline $\mathrm{Co}-\mathrm{S}-\mathrm{O} / \mathrm{NSCN}$ & 1.0 & 162 & $\begin{array}{c}20 \mathrm{~mA} \mathrm{~cm}{ }^{-2}, 40 \mathrm{~min} / \text { cycle for } \\
62 \mathrm{~h} \text { and } 20 \mathrm{~mA} \mathrm{~cm}^{-2}, 4 \mathrm{~h} / \text { cycle } \\
\text { for } 100 \mathrm{~h} \text {; voltage gap increased } \\
\sim 0.02 \mathrm{~V}\end{array}$ & $\begin{array}{l}\text { This } \\
\text { work. }\end{array}$ \\
\hline N-GCNT/FeCo-3 & 2.0 & 89 & $\begin{array}{c}20 \mathrm{~mA} \mathrm{~cm}^{-2}, 20 \mathrm{~min} / \text { cycle for } \\
27 \text { cycles; voltage gap increased } \\
\sim 0.03 \mathrm{~V}\end{array}$ & [16] \\
\hline NPMC-1000 & 0.5 & 55 & $\begin{array}{c}2,10 \text { min per cycle for } 180 \\
\text { cycles }(30 \mathrm{~h})\end{array}$ & {$[17]$} \\
\hline NCNF-1000 & 2.0 & 185 & $\begin{array}{c}10 \mathrm{~mA} \mathrm{~cm}^{-2}, 10 \mathrm{~min} / \text { cycle for } \\
500 \text { cycles; voltage gap } \\
\text { increased } \sim 0.13 \mathrm{~V}\end{array}$ & {$[18]$} \\
\hline NCN-1000-80 & 2.0 & 207 & $\begin{array}{c}10 \mathrm{~mA} \mathrm{~cm}^{-2}, 20 \mathrm{~min} / \text { cycle for } \\
1000 \text { cycles; no obvious voltage } \\
\text { decay }\end{array}$ & [19] \\
\hline $\mathrm{N}-\mathrm{CN} 9$ & 1.0 & 41 & $\begin{array}{c}10 \mathrm{~mA} \mathrm{~cm}{ }^{-2}, 10 \mathrm{~min} / \text { cycle for } \\
30 \text { cycles; voltage gap increased } \\
\sim 0.33 \mathrm{~V}\end{array}$ & {$[20]$} \\
\hline 3D-CNTA & 2.0 & 157 & $\begin{array}{c}10 \mathrm{~mA} \mathrm{~cm}^{-2}, 10 \mathrm{~min} / \text { cycle for } \\
240 \text { cycles; voltage gap } \\
\text { increased } \sim 0.14 \mathrm{~V}\end{array}$ & {$[21]$} \\
\hline $1100-\mathrm{CNS}$ & 2.0 & 151 & $\begin{array}{c}10 \mathrm{~mA} \mathrm{~cm}^{-2}, 11 \mathrm{~min} / \text { cycle for } \\
300 \text { cycles; voltage gap } \\
\text { increased } \sim 0.08 \mathrm{~V}\end{array}$ & {$[22]$} \\
\hline PS-CNS & N.A. & 231 & $\begin{array}{c}2 \mathrm{~mA} \mathrm{~cm}^{-2}, 12 \mathrm{~min} / \text { cycle for } \\
600 \text { cycles; no obvious voltage } \\
\text { decay }\end{array}$ & {$[23]$} \\
\hline Co/CoO@Co-N-C & 2.0 & 157 & $\begin{array}{c}10 \mathrm{~mA} \mathrm{~cm}^{-2}, 10 \mathrm{~min} / \text { cycle for } \\
100 \text { cycles; voltage gap } \\
\text { increased } \sim 0.19 \mathrm{~V}\end{array}$ & {$[24]$} \\
\hline $\mathrm{NiO} / \mathrm{CoN}$ PINWs & N.A. & 80 & $\begin{array}{c}3 \mathrm{~mA} \mathrm{~cm}{ }^{-2}, 10 \mathrm{~min} / \text { cycle for } 50 \\
\text { cycles; voltage gap increased } \\
\sim 0.25 \mathrm{~V}\end{array}$ & {$[25]$} \\
\hline $\mathrm{Ni}_{3} \mathrm{Fe} / \mathrm{N}-\mathrm{C}$ & 2.0 & N.A. & $\begin{array}{c}10 \mathrm{~mA} \mathrm{~cm}{ }^{-2}, 4 \mathrm{~h} / \text { cycle for } 105 \\
\text { cycles; voltage gap increased } \\
\sim 0.20 \mathrm{~V}\end{array}$ & {$[26]$} \\
\hline
\end{tabular}


CoZn-NC-700

MnO@Co-N/C

0.6

N.A.

$\mathrm{Co}_{3} \mathrm{O}_{4} / \mathrm{N}-\mathrm{rGO}$

$\mathrm{Fe}_{0.5} \mathrm{Co}_{0.5} \mathrm{O}_{\mathrm{x}} / \mathrm{NrGO}$

1.0

0.5

$\mathrm{Co}_{3} \mathrm{O}_{4} / \mathrm{N}-\mathrm{CNTAs}$

Co- $\mathrm{N}_{\mathrm{x}}-\mathrm{C}$

0.50

2.0

$\mathrm{CoS}_{\mathrm{x}} @ \mathrm{PCN} / \mathrm{rGO}$

$\mathrm{RuO}_{2}$-coated MCNAs

$1.5 \sim 2.0$

0.5
152

130.3

N.A.

86

N.A.

152

N.A.

N.A.

105
$10 \mathrm{~mA} \mathrm{~cm}{ }^{-2}, 10 \mathrm{~min} /$ cycle for 385 cycles; voltage gap increased $\sim 0.37 \mathrm{~V}$ 1900 cycles 75 cycles 60 cycles 108 cycles

$$
5 \mathrm{~mA} \mathrm{~cm}{ }^{-2}, 10 \text { min per cycle }
$$
for 100 cycles

$2 \mathrm{~mA} \mathrm{~cm}{ }^{-2}, 20 \mathrm{~min}$ per cycle for 180 cycles for 394 cycles

$$
100 \text { cycles }
$$

$10 \mathrm{~mA} \mathrm{~cm}^{-2}, 20 \mathrm{~min}$ per cycle for 90 cycles

\section{Reference}

(1) Liu, S.; Wang, Z.; Zhou, S.; Yu, F.; Yu, M.; Chiang, C. Y.; Zhou, W.; Zhao, J.; Qiu, J. Metal-Organic-Framework-Derived Hybrid Carbon Nanocages as a Bifunctional Electrocatalyst for Oxygen Reduction and Evolution. Adv. Mater. 2017, 29, 1700874, DOI 10.1002/adma.201700874.

(2) Zhong, H.-x.; Li, K.; Zhang, Q.; Wang, J.; Meng, F.-1.; Wu, Z.-j.; Yan, J.-m.; Zhang, X.-b. In situ anchoring of $\mathrm{Co}_{9} \mathrm{~S}_{8}$ nanoparticles on $\mathrm{N}$ and $\mathrm{S}$ co-doped porous carbon tube as bifunctional oxygen electrocatalysts. NPG Asia Mater. 2016, 8, e308, DOI 10.1038/am.2016.132.

(3) Aijaz, A.; Masa, J.; Rosler, C.; Xia, W.; Weide, P.; Botz, A. J.; Fischer, R. A.; Schuhmann, W.; Muhler, M. $\mathrm{Co} @ \mathrm{Co}_{3} \mathrm{O}_{4}$ Encapsulated in Carbon Nanotube-Grafted Nitrogen-Doped Carbon Polyhedra as an Advanced Bifunctional Oxygen Electrode. Angew. Chem. Int. Ed. 2016, 55, 4087-4091, DOI 10.1002/anie.201509382. 
(4) Mao, S.; Wen, Z.; Huang, T.; Hou, Y.; Chen, J. High-performance bi-functional electrocatalysts of 3D crumpled graphene-cobalt oxide nanohybrids for oxygen reduction and evolution reactions. Energy Environ. Sci. 2014, 7, 609-616, DOI 10.1039/c3ee42696c.

(5) Fu, G.; Chen, Y.; Cui, Z.; Li, Y.; Zhou, W.; Xin, S.; Tang, Y.; Goodenough, J. B. Novel Hydrogel-Derived Bifunctional Oxygen Electrocatalyst for Rechargeable Air Cathodes. Nano Lett. 2016, 16, 6516-6522, DOI 10.1021/acs.nanolett.6b03133.

(6) Jia, N.; Liu, J.; Gao, Y.; Chen, P.; Chen, X.; An, Z.; Li, X.; Chen, Y. Graphene-Encapsulated Co 9 S8 Nanoparticles on N,S-Codoped Carbon Nanotubes: An Efficient Bifunctional Oxygen Electrocatalyst. ChemSusChem 2019, 12, 3390-3400, DOI 10.1002/cssc.201900383.

(7) Fang, W.; Hu, H.; Jiang, T.; Li, G.; Wu, M. N- and S-doped porous carbon decorated with in-situ synthesized Co-Ni bimetallic sulfides particles: A cathode catalyst of rechargeable Zn-air batteries. Carbon 2019, 146, 476-485, DOI 10.1016/j.carbon.2019.01.027.

(8) Hu, W.; Wang, Q.; Wu, S.; Huang, Y. Facile one-pot synthesis of a nitrogen-doped mesoporous carbon architecture with cobalt oxides encapsulated in graphitic layers as a robust bicatalyst for oxygen reduction and evolution reactions. J. Mater. Chem. A 2016, 4, 16920-16927, DOI 10.1039/c6ta08103g.

(9) Zhang, Y.; Chao, S.; Wang, X.; Han, H.; Bai, Z.; Yang, L. Hierarchical $\mathrm{Co}_{9} \mathrm{~S}_{8}$ hollow microspheres as multifunctional electrocatalysts for oxygen reduction, oxygen evolution and hydrogen evolution reactions. Electrochim. Acta 2017, 246, 380-390, DOI 10.1016/j.electacta.2017.06.058.

(10) Xu, N.; Qiao, J.; Nie, Q.; Wang, M.; Xu, H.; Wang, Y.; Zhou, X.-D. $\mathrm{CoFe}_{2} \mathrm{O}_{4}$ nanoparticles decorated carbon nanotubes: Air-cathode bifunctional catalysts for rechargeable zinc-air batteries. Catal. Today 2018, 318 , 144-149, DOI 10.1016/j.cattod.2017.10.020

(11) Shui, H.; Jin, T.; Hu, J.; Liu, H. In Situ Incorporation Strategy for Bimetallic FeCo-Doped Carbon as Highly Efficient Bifunctional Oxygen Electrocatalysts. Chemelectrochem 2018, 5, 1401-1406, DOI 10.1002/celc.201800013.

(12) Bai, J.; Meng, T.; Guo, D.; Wang, S.; Mao, B.; Cao, M. Co. $\mathrm{S}_{8} @ \mathrm{MoS}_{2}$ Core-Shell Heterostructures as Trifunctional Electrocatalysts for Overall Water Splitting and Zn-Air Batteries. ACS Appl. Mater. Inter. 2018, 10, 1678-1689, DOI 10.1021/acsami.7b14997.

(13) Liu, X. X.; Zang, J. B.; Chen, L.; Chen, L. B.; Chen, X.; Wu, P.; Zhou, S. Y.; Wang, Y. H. A microwave-assisted synthesis of $\mathrm{CoO} @$ Co core-shell structures coupled with N-doped reduced graphene oxide used as a superior multi-functional electrocatalyst for hydrogen evolution, oxygen reduction and oxygen evolution reactions. J. Mater. Chem. A 2017, 5, 5865-5872, DOI 10.1039/c6ta10591b.

(14) Meng, T.; Qin, J.; Wang, S.; Zhao, D.; Mao, B.; Cao, M. In situ coupling of $\mathrm{Co}_{0.85} \mathrm{Se}$ and N-doped carbon via one-step selenization of metal-organic frameworks as a trifunctional catalyst for overall water splitting and $\mathrm{Zn}$-air batteries. J. Mater. Chem. A 2017, 5, 7001-7014, DOI 10.1039/c7ta01453h. 
(15) Cheng, Y.; Liao, F.; Shen, W.; Liu, L.; Jiang, B.; Li, Y.; Shao, M. Carbon cloth supported cobalt phosphide as multifunctional catalysts for efficient overall water splitting and zinc-air batteries. Nanoscale 2017, 9, 18977-18982, DOI 10.1039/c7nr06859j.

(16) Su, C.-Y.; Cheng, H.; Li, W.; Liu, Z.-Q.; Li, N.; Hou, Z.; Bai, F.-Q.; Zhang, H.-X.; Ma, T.-Y. Atomic Modulation of FeCo-Nitrogen-Carbon Bifunctional Oxygen Electrodes for Rechargeable and Flexible All-Solid-State Zinc-Air Battery. Adv.Energy Mater. 2017, 7, 1602420, DOI 10.1002/aenm.201602420.

(17) Zhang, J.; Zhao, Z.; Xia, Z.; Dai, L. A metal-free bifunctional electrocatalyst for oxygen reduction and oxygen evolution reactions. Nat. Nanotechnol. 2015, 10, 444-452, DOI 10.1038/nnano.2015.48.

(18) Liu, Q.; Wang, Y.; Dai, L.; Yao, J. Scalable Fabrication of Nanoporous Carbon Fiber Films as Bifunctional Catalytic Electrodes for Flexible Zn-Air Batteries. Adv. Mater. 2016, 28, 3000-3006, DOI 10.1002/adma.201506112.

(19) Jiang, H.; Gu, J.; Zheng, X.; Liu, M.; Qiu, X.; Wang, L.; Li, W.; Chen, Z.; Ji, X.; Li, J. Defect-rich and ultrathin $\mathrm{N}$ doped carbon nanosheets as advanced trifunctional metal-free electrocatalysts for the ORR, OER and HER. Energy Environ. Sci. 2019, 12, 322-333, DOI 10.1039/c8ee03276a.

(20) Zhang, C.; Zhang, G.; Li, H.; Chang, Y.; Chang, Z.; Liu, J.; Sun, X. Interfacial dehalogenation-enabled hollow N-doped carbon network as bifunctional catalysts for rechargeable Zn-air battery. Electrochim. Acta 2017, 247, 1044-1051, DOI 10.1016/j.electacta.2017.07.099.

(21) Wang, S.; Qin, J.; Meng, T.; Cao, M. Metal-organic framework-induced construction of actiniae-like carbon nanotube assembly as advanced multifunctional electrocatalysts for overall water splitting and $\mathrm{Zn}$-air batteries. Nano Energy 2017, 39, 626-638, DOI 10.1016/j.nanoen.2017.07.043.

(22) Pei, Z.; Li, H.; Huang, Y.; Xue, Q.; Huang, Y.; Zhu, M.; Wang, Z.; Zhi, C. Texturing in situ: N,S-enriched hierarchically porous carbon as a highly active reversible oxygen electrocatalyst. Energy Environ. Sci. 2017, 10, 742-749, DOI 10.1039/c6ee03265f.

(23) Shinde, S. S.; Lee, C. H.; Sami, A.; Kim, D. H.; Lee, S. U.; Lee, J. H. Scalable 3-D Carbon Nitride Sponge as an Efficient Metal-Free Bifunctional Oxygen Electrocatalyst for Rechargeable Zn-Air Batteries. ACS Nano 2017, 11, 347-357, DOI 10.1021/acsnano.6b05914.

(24) Zhang, X.; Liu, R.; Zang, Y.; Liu, G.; Wang, G.; Zhang, Y.; Zhang, H.; Zhao, H. Co/CoO nanoparticles immobilized on Co-N-doped carbon as trifunctional electrocatalysts for oxygen reduction, oxygen evolution and hydrogen evolution reactions. Chem. Commun. 2016, 52, 5946-5949, DOI 10.1039/c6cc02513g.

(25) Yin, J.; Li, Y.; Lv, F.; Fan, Q.; Zhao, Y. Q.; Zhang, Q.; Wang, W.; Cheng, F.; Xi, P.; Guo, S. NiO/CoN Porous Nanowires as Efficient Bifunctional Catalysts for Zn-Air Batteries. ACS Nano 2017, 11, 2275-2283, DOI 10.1021/acsnano.7b00417. 
(26) Fu, G.; Cui, Z.; Chen, Y.; Li, Y.; Tang, Y.; Goodenough, J. B. Ni ${ }_{3} \mathrm{Fe}-\mathrm{N}$ Doped Carbon Sheets as a Bifunctional Electrocatalyst for Air Cathodes. Adv. Energy Mate. 2017, 7, 1601172, DOI 10.1002/aenm.201601172.

(27) Wu, X.; Han, X.; Ma, X.; Zhang, W.; Deng, Y.; Zhong, C.; Hu, W. Morphology-Controllable Synthesis of Zn-Co-Mixed Sulfide Nanostructures on Carbon Fiber Paper Toward Efficient Rechargeable Zinc-Air Batteries and Water Electrolysis. ACS Appl. Mater. Inter. 2017, 9, 12574-12583, DOI 10.1021/acsami.6b16602.

(28) Chen, Y.-N.; Guo, Y.; Cui, H.; Xie, Z.; Zhang, X.; Wei, J.; Zhou, Z. Bifunctional electrocatalysts of MOF-derived Co-N/C on bamboo-like $\mathrm{MnO}$ nanowires for high-performance liquid- and solid-state $\mathrm{Zn}$-air batteries. J. Mater. Chem. A 2018, 6, 9716-9722, DOI 10.1039/c8ta01859f.

(29) Li, Y.; Zhong, C.; Liu, J.; Zeng, X.; Qu, S.; Han, X.; Deng, Y.; Hu, W.; Lu, J. Atomically Thin Mesoporous $\mathrm{Co}_{3} \mathrm{O}_{4}$ Layers Strongly Coupled with N-rGO Nanosheets as High-Performance Bifunctional Catalysts for 1D Knittable Zinc-Air Batteries. Adv. Mater. 2018, 30, 1703657, DOI 10.1002/adma.201703657.

(30) Wei, L.; Karahan, H. E.; Zhai, S.; Liu, H.; Chen, X.; Zhou, Z.; Lei, Y.; Liu, Z.; Chen, Y. Amorphous Bimetallic Oxide-Graphene Hybrids as Bifunctional Oxygen Electrocatalysts for Rechargeable Zn-Air Batteries. Adv. Mater. 2017, 29, 1701410, DOI 10.1002/adma.201701410.

(31) Wang, H. F.; Tang, C.; Wang, B.; Li, B. Q.; Zhang, Q., Bifunctional Transition Metal Hydroxysulfides: Room-Temperature Sulfurization and Their Applications in Zn-Air Batteries. Adv. Mater. 2017, 29, 1702327, DOI 10.1002/adma.201702327.

(32) Tian, W.; Li, H.; Qin, B.; Xu, Y.; Hao, Y.; Li, Y.; Zhang, G.; Liu, J.; Sun, X.; Duan, X. Tuning the wettability of carbon nanotube arrays for efficient bifunctional catalysts and $\mathrm{Zn}$-air batteries. J. Mater. Chem. A 2017, 5, 7103-7110, DOI 10.1039/c6ta10505j.

(33) Tang, C.; Wang, B.; Wang, H. F.; Zhang, Q. Defect Engineering toward Atomic Co- $\mathrm{N}_{\mathrm{x}}-\mathrm{C}$ in Hierarchical Graphene for Rechargeable Flexible Solid Zn-Air Batteries. Adv. Mater. 2017, 29, 1703185, DOI 10.1002/adma.201703185.

(34) Niu, W.; Li, Z.; Marcus, K.; Zhou, L.; Li, Y.; Ye, R.; Liang, K.; Yang, Y. Surface-Modified Porous Carbon Nitride Composites as Highly Efficient Electrocatalyst for Zn-Air Batteries. Adv. Energy Mater. 2018, 8, 1701642, DOI 10.1002/aenm.201701642.

(35) Guo, Z.; Li, C.; Li, W.; Guo, H.; Su, X.; He, P.; Wang, Y.; Xia, Y. Ruthenium oxide coated ordered mesoporous carbon nanofiber arrays: a highly bifunctional oxygen electrocatalyst for rechargeable $\mathrm{Zn}$-air batteries. J. Mater. Chem. A 2016, 4, 6282-6289, DOI 10.1039/c6ta02030e.

(36) Zhang, M.; Dai, Q.; Zheng, H.; Chen, M.; Dai, L. Novel MOF-Derived Co@N-C Bifunctional Catalysts for Highly Efficient Zn-Air Batteries and Water Splitting. Adv. Mater. 2018, 30, 1705431, DOI 10.1002/adma.201705431. 